\title{
Duloxetin als Ergänzung zum Beckenbodentraining
}

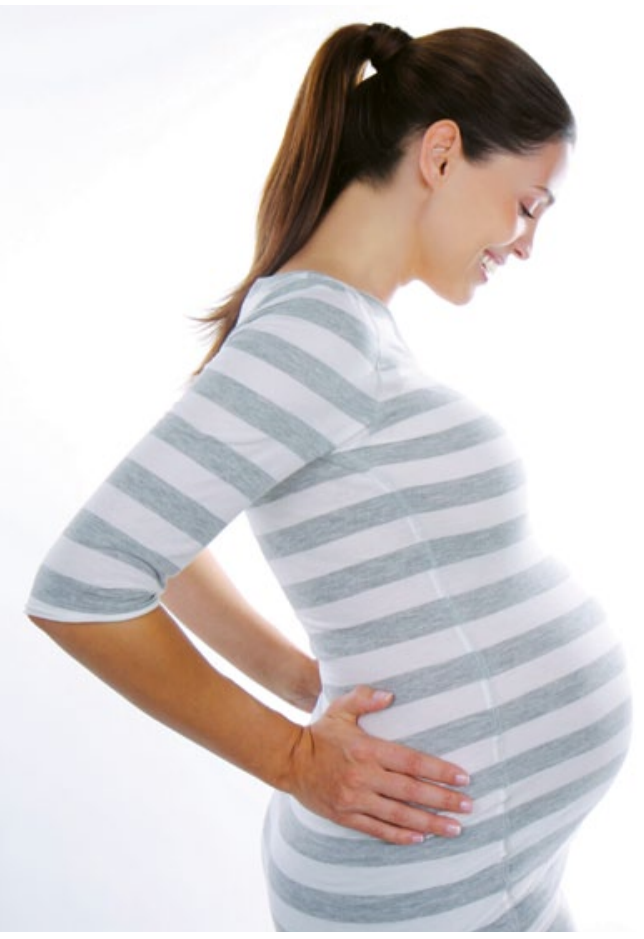

Nach Schwangerschaft und Geburt leiden viele Frauen - auch jüngere - an einer Belastungsinkontinenz.
- Schwangerschaft und Geburt können sich auf die Beckenbodenmuskulatur auswirken und eine Belastungsinkontinenz verursachen. „Dies liegt zumeist an den Läsionen muskulärer und bindegeweblicher Art durch die Geburt", erklärt Dr. Gert Naumann, Gynäkologe aus Mainz. Studien, wie die von Rortveit et al. mit mehr als 15.000 Teilnehmerinnen, bestätigen das: Der Anteil von Frauen allen Alters, die nach einer spontanen vaginalen Geburt unter Belastungsinkontinenz litten, lag bei $14,7 \%$. Nach einer Sectio trat bei 7,0\% der Frauen eine Belastungsinkontinenz auf. Zu beobachten war, dass sich mit dem Alter der Einfluss des Geburtsmodus auf das generelle Auftreten von Harninkontinenz verliert: So zeigte sich bei $28,6 \%$ der Frauen zwischen 50 und 64 Jahren, die im Laufe ihres Lebens eine Sectio hatten, eine Form von Harninkontinenz. Frauen, die jemals spontan vaginal entbunden hatten, litten vergleichbar oft, nämlich zu $30 \%$ unter Belastungs-, Drang oder Mischinkontinenz. Bei einer Belastungsinkontinenz nach der Entbindung kann schon früh eine erfolgreiche Behandlung der Symptome eingeleitet werden. Eine wichtige Rolle dabei spielt die Aufklärung der Frauen über das Behandlungsspektrum. Bereits während der Schwangerschaft ist es möglich, mit einem professionell angeleiteten Beckenbodentraining die Muskulatur zu stärken. „Drei bis sechs Monate nach der Geburt sind die meisten Frauen bereit, die Übungen wieder aufzunehmen", weiß Naumann. Mit dem Ende der Stillzeit kann auch Duloxetin (Yentreve ${ }^{\oplus}$ ) ergänzend zum Beckenbodentraining eingesetzt werden. „Gerade bei jungen Frauen mache ich immer wieder gute Erfahrungen mit dieser konservativen Kombinationstherapie", erklärt Naumann.

Dass Patientinnen mit Belastungsinkontinenz vor allem von der Kombination aus Duloxetin und physiotherapeutisch angeleitetem Beckenbodentraining profitieren hat auch die SUIT-Studie („stress urinary incontinence treatment ${ }^{\prime \prime}$ ) verdeutlicht.

Prägnante Übersichten über das passende Therapieschema, aktuelle Studien und zahlreiche weitere Hintergrundmaterialien finden sich auf www.Yentreve.de.

Nach Informationen von

Lilly, Bad Homburg

\section{Prostatakarzinom: rasche Senkung der Testosteronlevel entscheidend}

— In der Behandlung des fortgeschrittenen Prostatakarzinoms ist die Androgendeprivationstherapie (ADT) Standard. Die Testosteronspiegel der Patienten lassen sich mit Gonadotropin-Releasing-Hormon-Agonisten (GnRH-Agonisten) senken, zusätzlich ist eine kurzzeitige Gabe von Antiandrogenen zur Flare-up-Prophylaxe erforderlich. Im Gegensatz zu den Agonisten blockieren GnRHAntagonisten die Gonadotropinausschüttung umgehend und sorgen so dafür, dass Testosteron schnell gesenkt wird.

In der Zulassungsstudie konnte gezeigt werden, dass der GnRH-Blocker Degarelix (Firmagon ${ }^{\oplus}$ ) über einen Zeitraum von zwölf Monaten das Serumtestosteron senken kann und gleichzeitig keine Flare-Symptome auslöst. Prof. Thomas Keane, Charleston (USA), stellte aktuelle Daten der Fünf-Jahres-Erweiterungsstudie vor [Crawford ED et al. J Urol 2010]. Leuprorelin-Patienten aus dem Kontrollarm wurden erneut randomisiert und erhielten initial 240 mg Degarelix und nach einem Monat eine Erhaltungsdosis von entweder $160 \mathrm{mg}$ oder $80 \mathrm{mg}$. Patienten, die bereits im ersten Jahr Degarelix erhalten hatten, bekamen weiterhin dieselbe Dosis. Nach dem Wechsel auf Degarelix hatten die ehemaligen Leuprorelin-Patienten ein signifikant längeres progressionsfreies Überleben. Die Zeit, innerhalb der bei $25 \%$ der Patienten mit einem Ausgangs-PSA-Wert von > $20 \mathrm{ng} /$ $\mathrm{ml}$ ein PSA-Versagen oder Tod eintrat, betrug unter Degarelix 514 Tage, 211 Tage mehr als unter Leuprorelin (304 Tage; $p=0,01$ ). Die Langzeitdaten bestätigen laut Keane die Wirkung und Verträglichkeit von Degarelix und unterstützen seine Anwendung in der Erstlinien-ADT.

Dass Degarelix auch in der Behandlung von Symptomen der unteren Harnwege (LUTS) GnRH-Agonisten überlegen sein könnte, zeigte John Anderson, Sheffield, anhand einer Studie mit Patienten mit lokal fort- geschrittenem Prostatakarzinom und zusätzlichen LUTS. So konnte unter Degarelix nach zwölf Wochen der internationale Prostata-Symptomen-Score (IPSS) der Betroffenen deutlicher gesenkt werden als unter Goserelin plus Antiandrogen (11,6 vs. 7,4; $\mathrm{p}=0,06)$. Auch die Prostatagröße nahm stärker ab $(41,8 \%$ vs. $24,6 \% ; p=0,03)$. Untermauert werden diese Daten durch eine Verbesserung des maximalen Harnfluss $\left(\mathrm{Q}_{\max }\right)$ und der Lebensqualität.

„Die Wirkung von GnRH-Agonist plus Antiandrogen ist nicht äquivalent zu der von GnRH-Blockern bezüglich Testosteron-, FSHund PSA-Kontrolle", fasste Anderson die Studienergebnisse zusammen

slx

Symposium "New diagnostic and treatment options for prostate cancer that will change your practice" im Rahmen des EAUKongresses, Wien, 20. März 2011

Veranstalter: Ferring, Kiel 